\title{
Breast cancer, primary peritoneal malignant mixed mullerian tumor and fallopian tube carcinoma: incidental concomitant malignancies or evidence for a new genetic cancer predisposition syndrome?
}

\author{
Estanislao, GL, Campos, RA
}

The Medical City, Metro Manila, Philippines

\begin{abstract}
This is a case report on a 72 year old postmenopausal G4P4(4-0-0-4), known case of stage I breast cancer, s/p modified radical mastectomy, maintained on Anastrozole, with no history of chemotherapy or radiotherapy. She has a family history of breast, cervical and kidney cancer. One year later she was diagnosed with synchronous primary peritoneal malignant mixed mullerian tumor (MMMT) and high grade serous fallopian tube carcinoma. While her BRCA status is unknown, recent studies have suggested the existence of another genetic cancer predisposition syndrome linking breast cancer and MMMT. While breast cancer generally has a good prognosis when managed early, MMMT is a high grade tumor with a poor prognosis. Because of this, discovery of such a hereditary link between breast cancer and MMMT impacts not only individuals but entire families as well. This link has been previously proposed but largely ignored. This case provides further support to this theorized gene.
\end{abstract}
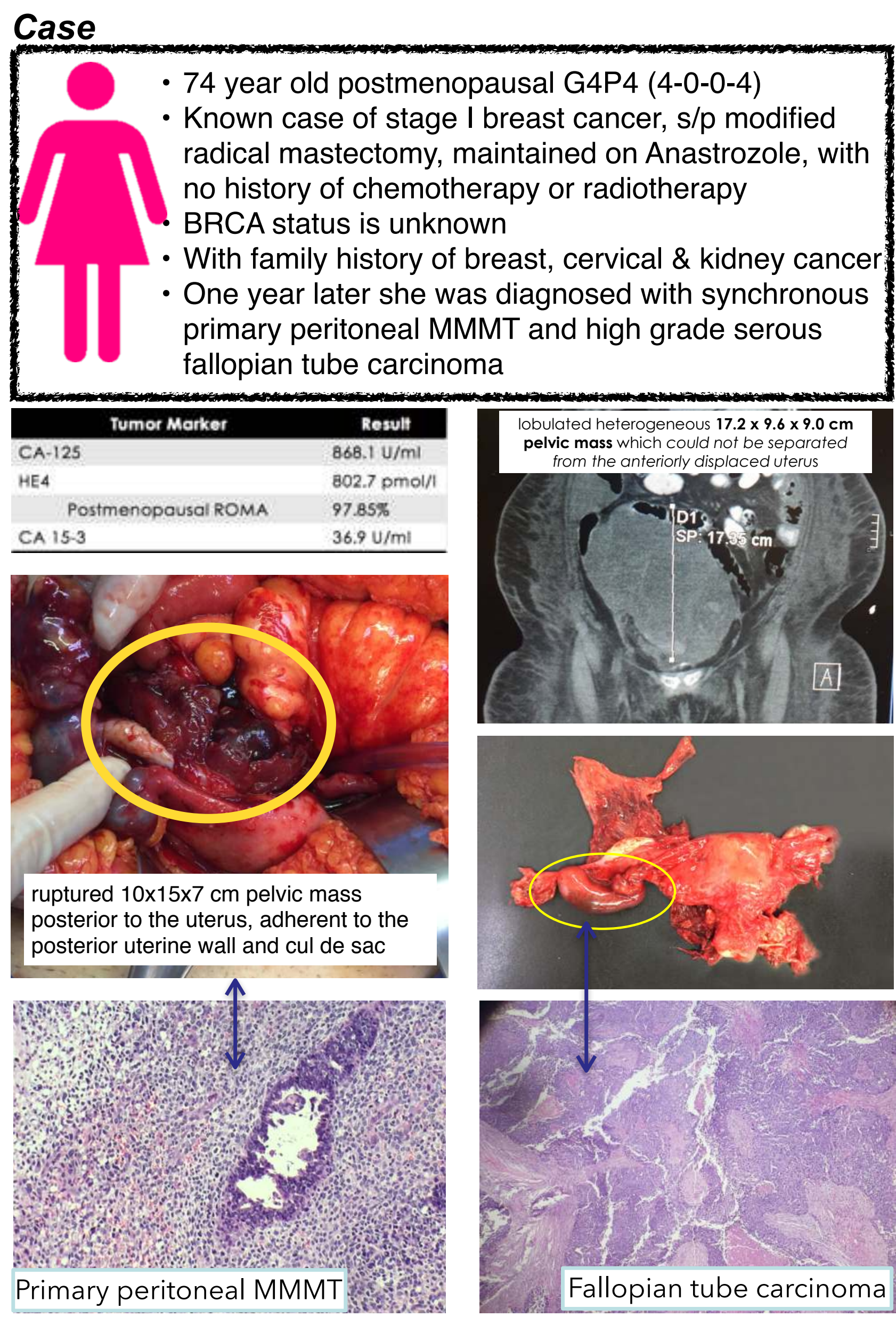

Since 1955, there have been 40 case reports of women with concomitant peritoneal malignant mixed mullerian tumor and other malignancies.
This review of case reports showed that extragenital MMMT usually occurs in postmenopausal women, commonly presents with abdominal pain, and has poor prognosis. These demographic and clinical characteristics are consistent with our patient. A number of the cases were associated with synchronous or metachronous malignancies, including that of Mullerian duct origin (ovarian tumors, endometrial cancer, cervical adenocarcinoma), colonic carcinomas and peritoneal serous carcinoma. However, none of them were noted to have occurred with breast cancer and fallopian tube carcinoma. Association with radiation and Tamoxifen therapy has been repeatedly reported in literature. However, there have been no studies linking MMMTs with aromatase inhibitors such as Anastrozole.

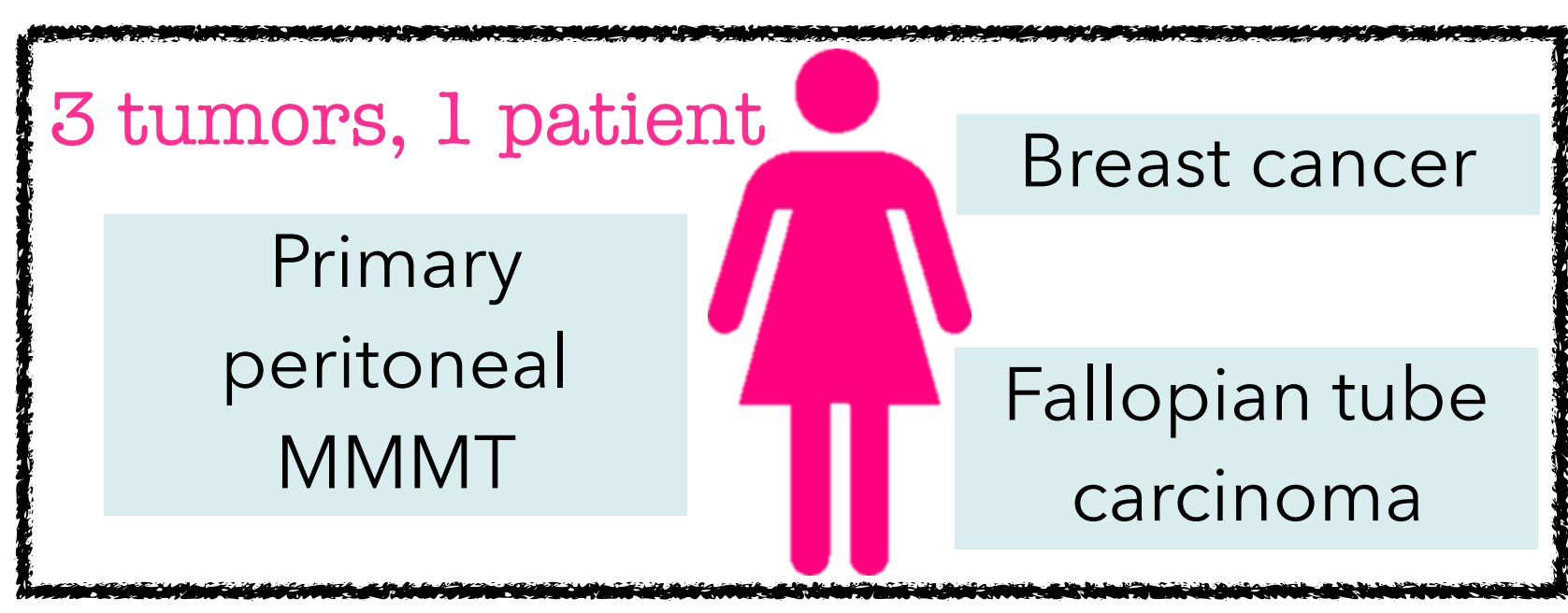

Where did it begin?

Scenario Fallopian tube high grade serous carcinoma

1 metastasised to the peritoneum and underwent sarcomatous metaplasia

Scenario Carcinomatous component of primary

2 peritoneal MMMT metastasised to the FT

\section{Scenario Genetic predisposition to develop breast \\ 3 cancer, primary peritoneal MMMT and fallopian tube carcinoma}

The likelihood of one gene directing the manifestation of these different primary tumors is strengthened by the recent paradigm shift that fallopian tube, peritoneal and ovarian cancers "are all of Mullerian origin and could therefore be regarded as a single disease entity." In 2003, a woman who had a strong family history of both breast and ovarian cancer was diagnosed with bilateral breast cancer and ovarian MMMT. She was not a BRCA1 or BRCA2 mutation carrier. It was suggested that there exists a different genetic mutation, which they dubbed "BRCA3." Similarly, a recent study published in March 2018 showed that $62.5 \%$ of patients with ovarian MMMT had metachronous or synchronous breast cancer. None were positive for BRCA1 or BRCA2 mutation.

The case of this patient is rare and practically unheard of in our local setting. However, she may just be an addition to a growing population of women affected by both breast cancer and MMMT. It is time we strongly consider the possibility of a new syndrome, which has been proposed but largely ignored. MMMT is an aggressive tumor and has a poor prognosis, such that even if breast cancer is detected and managed early, as in this patient, if one is positive for this theorized gene, the patient may still be lost to this high grade tumor. Putting effort into discovering this theorized gene will allow us to offer genetic counseling and consider life-saving prophylactic bilateral salpingooophorectomy with or without hysterectomy. 